\title{
LA INCAPACIDAD MAYÉUTICA DEL ARTE EN LA SOCIEDAD PLATÓNICA
}

\author{
Jorge Tomás García*
}

\begin{abstract}
RESUMEN
La relación entre Platón y el arte de su tiempo es un tema bien conocido desde hace décadas. Si bien parece claro que Platón estableció una definición perenne sobre qué es el arte y su proceso creativo en la antigua Grecia, sin embargo debemos prestar atención a la función que para Platón el arte tenía en su sociedad ideal. ¿Para qué el arte? En su proyecto educativo - basado en la mayéutica como eje fundamental del aprendizaje - el arte está sometido al dictamen y censura de las demás ciencias empíricas. Este artículo pretende evaluar la verdadera relevancia educativa que el proceso artístico tenía para Platón a través de un análisis crítico de la sociedad planteada por el filósofo ateniense.
\end{abstract}

Palabras clave: Platón. Arte. Pintura. Educación. Mayéutica. Censura.

\begin{abstract}
The relationship between Plato and the art of his time is a well known subject for decades. While it seems clear that Plato established a perpetual definition of what is art and the creative process in ancient Greece, however we must pay attention to the role that art had in Plato's ideal society. Why art? In its education process - based on maieutic as a fundamental learning motive - art is a subject to opinion and censorship of other empirical sciences. This article aims to evaluate the true educational relevance that Plato had artistic process through a critical analysis of society presented by the Athenian philosopher.
\end{abstract}

Keywords: Plato. Art. Painting. Education. Maieutic. Censorship.

\footnotetext{
* Doctor en Historia del arte y licenciando en Filología Clásica. Especializado en la Estética de la pintura griega clásica y su relación con la estética de Platón y Aristóteles. Profesor interino del Departamento de Historia del Arte en la Universidad de Murcia (España). E-mail: jtg.jorge@gmail.com
} 


\section{Introducción}

En la metáfora de la caverna del libro VII de la República de Platón es donde se tiene que analizar la verdadera capacidad social del arte para el filósofo ateniense. Para Platón lo que el arte tenía de valía o no es tan sólo apreciable a la luz de la sociedad y de su esquema compositivo. En ese proceso relativo a la mayéutica socrática que los individuos deben seguir para liberarse de las sombras de la caverna, el arte ocupa un lugar discutido y discutible. El concepto de $\pi \alpha 1 \delta \varepsilon i ́ \alpha$, normalmente traducido como "educación", abarca un sentido mucho más amplio de lo que hoy en día podemos entender por "educar". Para Heidegger, la única solución al

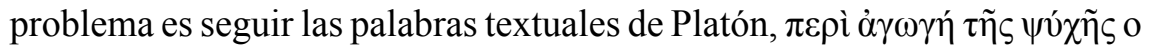
"dirigir la reversión del hombre entero a su esencia". La verdadera meta del ciudadanoes la delograruna existenciadesencadenadadelas sombras, através

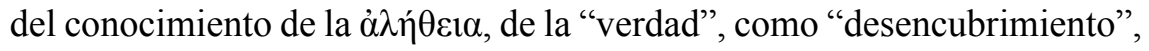
como contemplación de la realidad más verdadera y esencial después de haber alcanzado la verdad que arroja las $\varepsilon i \delta \delta \eta$. De esta manera, el ciudadano tiene que conseguir una conversión del alma entera para poder ascender hasta una visión nítida, clara y verdadera de las cosas. Pero, ¿qué función tiene el arte, si es que tiene alguna, en este proceso fundamental de la filosofía platónica? Para poder responder a esta pregunta capital primero tenemos que analizar qué entiende Platón por arte dentro de la sociedad utópica planteada en algunos de sus diálogos de madurez. La respuesta

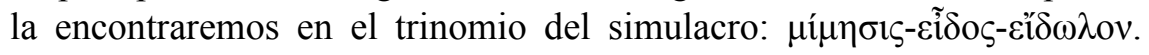

\section{La definición del concepto $\mu$ í́ simulacro}

El contexto dramático es el adecuado para realizar una primera

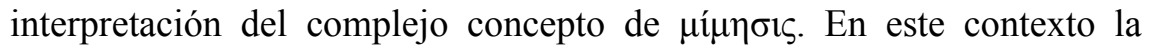
$\mu$ í $\eta \sigma ı s$, lejos del tradicional significado que posteriormente utilizará Platón en sus diálogos como principal arma arrojadiza contra el valor esencial del arte. La danza y la música se entendían como artes mayores con su propio lenguaje y, en ocasiones, abarcaban a las artes plásticas de la pintura o la escultura dentro de su propia teoría del arte se entiende como 
"representación" de movimientos dentro de los espectáculos de danza ${ }^{1}$, en el sentido de "Darstellung" y "Ausdruck", y no como "Nachahmung”, "imitación"'. Entonces, en el drama griego arcaico el concepto servía para significar "representar a través de la danza" ("durch Tanz zur Darstellung bringen"). El origen semántico de este significado arcaico se encuentra en el grupo de palabras derivadas de $\mu \tilde{i} \mu \varsigma^{3}$, seguramente con origen en $\mathrm{Sicilia}^{4}$, lejos del tradicional significado que posteriormente utilizará Platón en sus diálogos como principal arma arrojadiza contra el valor esencial del arte ${ }^{5}$. Hasta Platón, por lo tanto, la utilización de $\mu$ í $\eta$ бıৎ no implicaba el sentido de copia fiel de la realidad y reproducción exacta de lo contemplado por el artista ${ }^{6}$.

En el siglo V a.C. se produce una variedad de usos y significados del concepto ${ }^{7}$, si bien se mantiene el sentido general relacionado con la raíz

1 KOLLER, H. Die Mimesis in der Antike: Nachahmung, Darstellung, Ausdruck. Berna: Francke, 1954. En la obra de Koller se realiza una interpretación de la $\mu$ í $\eta$ $\sigma ı \varsigma$ desde el libro III de la República, del concepto de "danza, baile", ("Tanzes"), en Leyes, o del lenguaje, ("Sprache"), en el Crátilo. Además analiza detenidamente el libro X de la República, y la definición de "música" en la Política de Aristóteles; GOLDEN, L. Plato's Concept of Mimesis. BritJAesthet, n. 15, v. 2, 1975, p. 118-131.

2 SOBREVILLA, D. La Estética de la Antigüedad: Estudios sobre lo bello y el arte en el pensamiento de Platón, Aristóteles, Cicerón y Plotino. Valencia: Universidad de Carabobo, 1981. p. 21 y ss.; VERNANT, J. P. Image et apparence dans la théorie platonicienne de la mimesis. Journal de psycologie normale et pathologique, v. 2, 1975, p. 133-160; FLASHAR, H. Die Klassizistische Theorie der Mimesis. Le Classicisme a Rome, v. 25, 1978, p. 79-111.

3 KEULS, E. Plato and Greek Painting. Leiden: Brill, 1978. Realiza una revisión del significado de $\mu$ í $\eta_{\eta} \_\varsigma$ en el contexto de la pintura griega y analiza la tesis de Koller.

4 SÖRBOM, G. Mimesis and Art: Studies in the Origin and Early Development of an Aesthetic Vocabulary. Uppsala: Svenska Bökforlaget, 1966.

5 MORAUX, P. La "Mimesis" dans les théories anciennes de la danse, de la musique et de

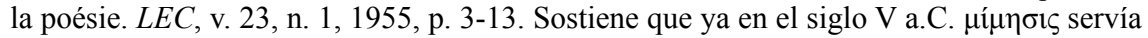
para designar cualquier tipo de representación artística y no sólo la danza o música.

6 DE ANGELI, S. Mimesis e Techne. QUCC, v. 57, 1988, p. 30: "La acción del mimo no implicaba una realización de fidelidad y perfecta identidad con el modelo en la imitación, más bien la se trataba de la capacidad de realizar una imagen expresiva gracias a la elección de una cualidad característica, del mismo modo la mimesis artística designa una acción centrada en la representación expresiva, a través de la elección de aspectos significativos".

7 Himno Délico a Apolo (1.163), Teognis (370), Esquilo (Cho. 564), Píndaro (Pítacas 
$\mu u$ - como la imitación a través de la apariencia externa de movimientos humanos o animales por seres humanos ${ }^{8}$. Platón no es ajeno a todos estos significados de $\mu$ í $\mu$ ¡ı $\varsigma$ y, por lo tanto, actúa como heredero de una tradición que le pertenece $\mathrm{y}$, a la vez, como creador de un nuevo escenario conceptual. La $\mu$ í $\eta$ $\sigma \varsigma \varsigma$ en Platón es un gran principio formal que penetra en todos los aspectos de la existencia, como aparece en Crátilo (423c424b), (426c-427d) y Leyes (668a-b), siendo entonces una herramienta para investigar diversas formas de la realidad 9 . No se configura como un concepto fijo y único para ciertas formas de lenguaje o realidad, sino que se sirve de él para expresar una relación dinámica entre el mundo y las apariencias, bien a través de la pintura, o de la escultura, o de la danza ${ }^{10}$, como aproximación dialéctica a la realidad ${ }^{11}$. Esta visión de la realidad como estructura jerárquica, por la que se puede ascender o descender según el nivel de falsedad o pureza ontológica, tuvo mucho éxito posteriormente ligado al significado de $\mu$ í $\mu \eta \iota^{12}$.

La representación de la imagen como simulacro, ě́ $\delta \omega \lambda o v^{13}$, es la tarea que desempeña el artista imitativo en la sociedad griega ${ }^{14}$. Incapaz de

12.21, frag. 94b Snell y frag. 107a Snell), Aristófanes (Las ranas 108-09), Eurípides (Ion 1429), Heródoto (3.37).

8 HalliwelL, S. The Aesthetics of Mimesis: Ancient Texts and Modern Problems. Princeton University Press, 2002. p. 19 y ss.

9 MCKEON, R. Literary Criticism and the Concept of Imitation in Antiquity. ModPhilology, v. 34, n. 1, 1936, p. 1-35.

10 MURRAY, P. Plato on Poetry. Ion; Republic 376e-398b9; Republic 595-608b10. Cambridge University Press, 1996, p. 3-6.

11 VERDENIUS, W. J. Mimesis. Plato's Doctrine of artistic imitation and its meaning to us. Leiden: Brill, 1949.

12 Para ver el legado de la teoría de la mímesis de Platón en los siglos posteriores: BOYD, J. D. The Function of Mimesis and Its Decline, Cambridge, 1968, p. 5 y ss; AUERBACH, E. Mímesis: La representación de la realidad en la literatura occidental. México: Fondo de Cultura Económica, 1987, p. 522 y ss.

13 Las principales referencias a $\varepsilon i \delta \omega \lambda$ ov se encuentran en Gorg. 463d2, 463e4, Rep. 382b10, 386d5, 443c4, 586c4, 587d6, 598b8, 599a7, Theaet. 150c2, 151c3, 191d9, 208c5, Phaedr. 250d5, 255d8, 276a9, Soph. 239d4, 240a5, 264c12, 266c6, 266d4, Polit. 286a1, Leg. 830 b8.

14 MÁSMELA, C. Dialéctica de la imagen: Una interpretación del Sofista de Platón. Barcelona: Antropos Editorial, 2006, p. 14. El autor contextualiza de manera excelente el 
llegar a la esencia real de los objetos, el artista se contenta con ofrecer una

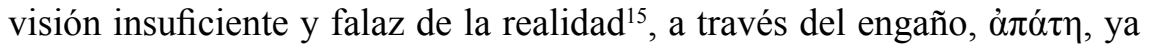

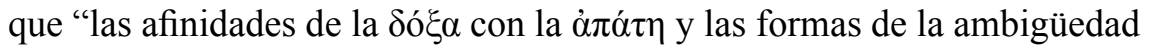
pueden encontrar su confirmación en determinadas significaciones fundamentales de la $\delta o ́ \xi \alpha "$. Es en el Sofista, Platón codifica y establece las dos opciones posibles para un artista de su tiempo ${ }^{16}$. Por una parte, la

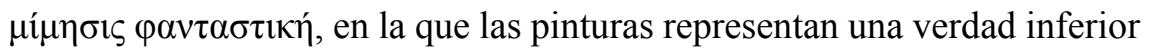
con las proporciones y distancias manipuladas por parte del artista para crear una sensación de ilusión y engaño, de manera que los espectadores son incapaces de juzgar la validez de la obra (Soph. 234c4, d4, e5-6). Por

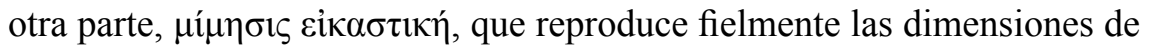
la obra en largo, alto y ancho (Soph. 235d7-8). La relación entre la "copia

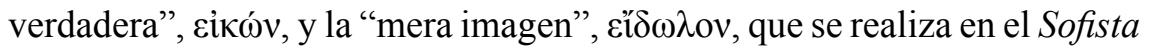
es compatible con la diferencia que se establece en la República entre

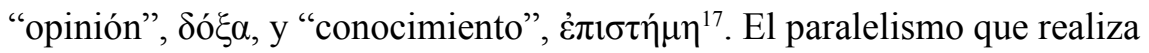
Platón entre el sofista y el artista es total en este punto; los identifica a los dos como creadores de imágenes ilusionistas, $\varphi \alpha v \tau \alpha ́ \sigma \mu \alpha \tau \alpha^{18}$, que creaban en el espectador una falsa creencia de realidad, produciendo simplemente simulacros. Un ejemplo de este tipo de simulacro es la pintura de sombras

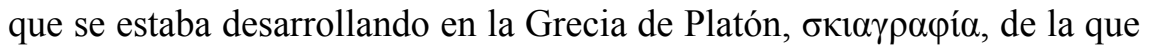
tratará posteriormente en República (602a).

discurso platónico acerca de la imagen, situando los posteriores hitos filosóficos en torno a este problema. La clasificación de las diferentes concepciones filosóficas que realizo están basadas en su original planteamiento.

15 PANOFSKY, E. Idea: Contribución a la historia de la teoría del arte. Madrid: Cátedra, 1977 , p. 15.

16 Ver especialmente NOTOMI, N. The Unity of Plato's Sophist: Between the Sophist and the Philosopher. Cambridge: Cambridge University Press, 1999.

17 LYONS, J. Structural Semantics: An Analysis of Part of the Vocabulary of Plato. Oxford: Blackwell, 1972. p. 194 y ss. Enumera una serie de sinónimos que Platón utiliza para referirse a la $\tau \dot{\chi} \chi v \eta$ en el Sofista y en el Político. Para el significado y el uso de $\mu$ í $\mu \eta \sigma ı$ en el Sofista, ver PHILIP, J. A. Mimesis in the Sophistes of Plato. TAMPhilolAssoc, v. 92, 1961, p. 453-468.

18 DELEUZE, G. Platón y el simulacro. In: Lógica del sentido, Barcelona, 2005, p. 295309. (Ed.original: Logique du sens. Paris: Ed. De Minuit, 1969). 
El sofista desprecia la imitación correcta de la naturaleza para servirse de una imitación conjetural cercana a la $\delta o ́ \xi \alpha$. Si bien ambos,

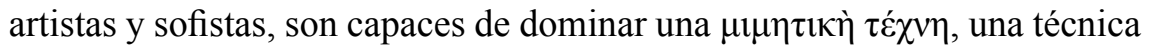
mimética, ninguno de los dos hace el uso requerido por Platón en su planteamiento social. La lucha de Platón contra sofistas se hacía todavía más complicada debido a la fama que tenían en la sociedad, ya que eran considerados como los depositarios de la verdad divina, herederos de la sabiduría poética de Homero y Hesíodo ${ }^{19}$. La distancia con los filósofos es considerable desde el punto de vista ontológico y epistemológico. Junto al sofista y al artista, es necesario analizar la opinión de Platón sobre los poetas, para poder finalizar con una conclusión final coherente que abarque todos los puntos de vista.

\section{La transmisión y la recepción del mensaje}

Efectivamente, los poetas son objeto de crítica y manipulación para el filósofo. En el Ion y en el Fedro se encuentran las críticas principales. En ambos diálogos el concepto capital es el de entusiasmo poético,

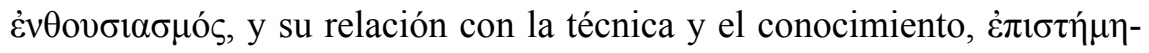
$\tau \varepsilon ́ \chi v \eta$. La no utilización de un método dialéctico por parte de las artes imitativas, y la carencia de $\tau \dot{\varepsilon} \chi \vee \eta$ por parte de los poetas, centran el diálogo de juventud $I{ }^{20}$. A partir de la crítica a los rapsodas incluye también a otros artistas como los escultores o algunos músicos (532e-533a), a los que no considera un buen ejemplo para el modelo de conducta adecuado y requerido para la ciudad, ya que su actividad consiste en recitaciones y

19 Las referencias a Homero en las obras de Platón son numerosas, variando el tratamiento y la intensidad de las críticas según cada obra: en GRISWOLD, C. The Ideas and the Criticism of the Poetry in Plato's Republic, Book 10. JHistPhilos, v. 19, n. 2, 1981, p. 135150 analiza el problema llegando a la siguiente conclusión: en el libro X Rep. cita a Homero y Hesíodo pero no nombra sus obras, en los libros II-III de la Rep. ataca más a la Ilíada que a la Odisea (libro II: 7 veces Ilíada- 2 Odisea, libro III: 25 Ilíada- 8 Odisea).

20 VERDENIUS, W. J. L'Ion de Platon. Mnemosyne, v. 11, 1943, p. 233-262; LADRIERE, C. The Problem of Plato's Ion. JAesthetArtCritic, v. 10, n. 1, 1951, p. 26-34; FLASHAR, H. Der Dialog Ion als Zeugnis platonischer Philosopie. Berlin: Akademie-Verlag, 1958. p. 25 y ss.; MOORE, J. D. The Dating of Plato's Ion. GreekRomanByzantin, v. 15, n. 4, 1974, p. 421-439. 
representaciones de recreaciones imitativas. Como se puede comprobar, el problema de la $\mu$ í $\eta$ $\sigma 1 \varsigma$ sigue estando presente en todo el discurso de Platón. La carencia en el conocimiento del objeto artístico tratado hace que los trabajos realizados por los poetas imitativos sean didácticamente inútiles para la formación del espíritu de los jóvenes griegos, que actúan como último eslabón en la cadena del entusiasmo poético al ser la audiencia receptora del mensaje poético ${ }^{21}$. El poeta, dominado por un arrebato interior, no se sirve de ninguna técnica racional o dialéctica cuando crea su obra, partiendo de la noción griega de $\pi$ oínoıs. El poeta se encuentra "lleno de

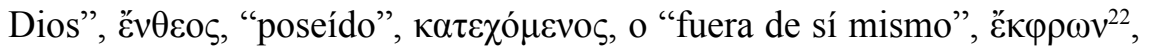
y de esta manera nunca podía llegar a poseer un verdadero conocimiento ${ }^{23}$.

En el Ion la Musa designa la presencia divina que envuelve al poeta

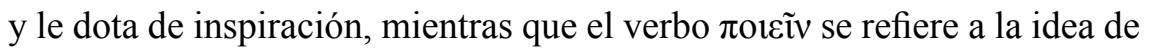
actividad creativa. Platón fue el primero que rompió con la idea arcaica del conocimiento relacionado con la presencia inspiradora de unas divinidades que alientan al poeta en el momento de creación poética y artística. También el filósofo Demócrito de Abdera (siglo IV a.C.) relacionó la creación poética con una reacción atómica del cuerpo ${ }^{24}$. La definición que Platón hace del poeta, deja bien claro que abandona cualquier esperanza de utilidad para su Estado Ideal, a no ser que fuera capaz de adaptarse a ciertas prerrogativas básicas. La ilegitimidad del poeta, una vez que ha quedado claro que compone por un impulso de la naturaleza divina ${ }^{25}$, le aleja de cualquier responsabilidad cívica en la ciudad, en la que el filósofo

21 GRIFFIN, J. The Social Function of Attic Tragedy. CQ, v. 48, n. 1, 1998, p. 39-61; DAVIDSON, J. Greek Drama: image and audience(s). BIClassStud, v. 48, 2005, p. 1-15.

22 Leg. (682a), (719c), Men. (99c), Apol. (22b-23e), Ion (533e-4e).

${ }^{23}$ DODDS, E. R. The Ancient Concept of Progress and other Essays on Greek Literatura and Belief. Oxford: Clarendon Paperbacks, 1973 p. 117 y ss.; TIGERSTEDT, E. N. Furor Poeticus: Poetic Inspiration in Greek Literature before Democritus and Plato. JHistIdeas, v. 31, n. 2, 1970, p. 163-178.

24 El concepto que tenían los pre-socráticos sobre la "inspiración" fue estudiado por DELATTE, A. Les conceptions de l'enthousiasme chez les philosophes présocratiques. Paris: Les Belles Lettres, 1934.

25 BABUT, D. Sur la notion d' Imitation dans les doctrines esthétiques de la Gréce classique. $R E G$, v. 98,1985 , p. $72-92$. 
debe ser el educador capaz de convertir el alma de los ciudadanos a través

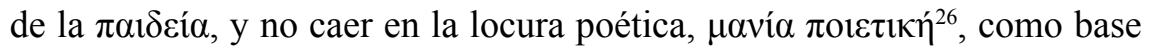
de la educación infantil.

\section{La utilidad de la belleza}

Los objetos resultantes de estos procesos creativos pueden ser calificados como bellos por parte de la sociedad y de los receptores de dichos objetos. La cualidad de la belleza es intrínseca en Platón a su función en la sociedad, pues ¿cómo iba a ser bello un objeto inútil o incapaz de producir enseñanza y conocimiento en el ciudadano? En el Hipias Mayor, diálogo que centra la discusión sobre la belleza, no se ofrece una solución estable y final al problema ${ }^{27}$. Esta belleza aporética tiene diversos matices:

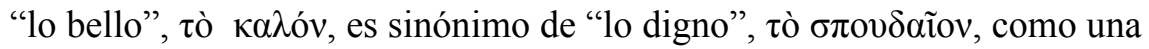

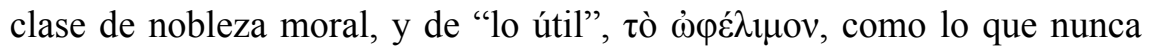
resulta superfluo. Hasta en cuatro se pueden dividir las definiciones que hace del concepto: la belleza como lo decoroso o favorecedor (Hip.mai. 296e2) la belleza como lo útil (Hip.mai 295e5) o adecuado (Hip.mai 294e5) y efectivo, la belleza como aquello que resulta adecuado para conseguir el bien y la belleza como lo que procura placer (Hip.mai. 303c8) a través de los sentidos más distinguidos (la vista y el oído). Este es el camino material para llegar a la posible contemplación de la belleza, de una belleza al alcance de los sentidos, de lo cotidiano y de la sociedad, pero una belleza

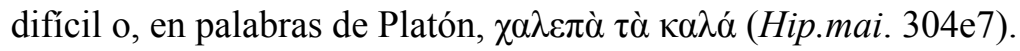

El otro camino es el camino trascendente. Si al camino material

${ }^{26}$ Para el significado de $\mu \alpha v i ́ \alpha$ ver VERDENIUS, W. J. Der Begriff der Mania in Platons Phaidros. ArchivGeschPhilos, 44:2, 1962, p. 133-151.

27 TARRANT, D. The Hippias Major. Cambridge, 1928; MALCOM, J. On the Place of the Hippias Major in the Development of Plato's Thought. ArchivGeschPhilos, vol. 50, n. 3, 1968, p. 189-195; WOODRUFF, P. Socrates and Ontology: The Evidence of the Hippias Major. Phronesis, v. 23, n. 2, 1978, p. 101-117; MORGAN, M. The Continuity Theory of Reality in Plato's Hippias Major. JHistPhilos, v. 21, n. 2, 1983, p. 133-158; SOLMSEN, F. Parmenides and the Description of Perfect Beauty in Plato's Symposium. AMJPhilology, v. 92, n. 1, 1971, p. 62-70; LIMINTA, M.T. Il problema della Belleza in Platone. Analisis e interpretazioni dell “Ippia Maggiore”. Milán: Vita e Pensiero, 1998. 
se accede a través de los sentidos, tal y como aparece en el Hipias Mayor, Filebo, para acceder al camino trascendente hay que servirse del intelecto y de la inteligencia. En el Banquete se traza el discurso sobre la belleza trascendente ${ }^{28}$. El amor en el discurso de Diotima se presenta como un $\delta \alpha i ́ \mu \omega v$, o espíritu, y la belleza está encuadrada entre los conceptos "verdad, bondad y belleza". En el fragmento que trata sobre el tema (210212), la belleza no se centra únicamente en los cuerpos o los objetos, sino que se amplía hasta llegar al sentimiento casi religioso de la filosofía que se desarrolla en el Banquete de Platón. En este texto, el filósofo ateniense extiende el significado tradicional del adjetivo $\kappa \alpha \lambda$ ós, incluyendo ahora la belleza de los conceptos abstractos e inaccesibles a la experiencia. De esta manera, la tradicional belleza física, ligada a los objetos y al mundo de los sentidos, queda totalmente devaluada frente a esta nueva concepción intelectual, que depende de su distancia con la Idea de lo Bello para catalogar el grado de belleza y verdad existente ${ }^{29}$. Por lo tanto, la búsqueda de esta belleza se realiza mediante el "E $\omega \varsigma$, ya que se le atribuye a Eros todas las cualidades bellas y, por encima de todas, la de la Belleza. En algunos pasajes del Fedro (250b-c), la visión de la Belleza romántica de Platón se expresa como "el arte como amor".

\section{$5 \mathrm{El}$ arte en su contexto}

El proceso de la mayéutica planteado por Platón tenía como base la justa y proporcionada utilización de estos conceptos teóricos. Tanto la

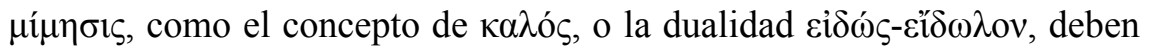

28 HUBER-ABRAHAMOWICZ, E. Das Problem der Kunst bei Platon. Winterthur: Keller, 1954. p. 9-14. analiza el significado filosófico del concepto de belleza relacionando la visión del Banquete con algunos fragmentos del Timeo, 29a, 30a, en el segundo capítulo "Die philosophische Bedeutung des Schönen"; WALTER, J. Die Geschichte der Ästhetik im Alterum. Hildesheim: Georg Olms, 1967. p. 202 y ss. "Kosmetische Elemente des Schönen"; BAEUMLER, A. Ästhetik. Berlin: Oldenbourg, 1934, p. 3-17, "Die Idee des Schönen".

29 La relación que se establece en el diálogo entre Sócrates y la belleza ha sido analizada por EDMONDS, R. G. Socrates the Beautiful: Role Reversal and Midwifery in Plato's Symposium. TAMPhilolAssoc, v. 130, 2000, p. 261-285; LESHER, J. H. The Afterlife of Plato's Symposium. ClassicalJ, v. 100, n. 1, 2004, p. 75-87. 
ser interpretadas a la luz de una sociedad particular, de una sociedad cerrada. K. Popper sentó las bases de la interpretación historicista de la obra de Platón ${ }^{30}$. Salvando los matices de la obra de Popper que se pueden considerar criticables o mejorables, su análisis es válido para comprender la sociedad platónica y, a partir de este análisis, ubicar al arte en su justa medida. Platón construyó teóricamente una "utopía" y una "eutopia"31. Un no lugar y un buen lugar, en 592a11 de la República se reafirman su proyecto teórico, el cual no puede existir en ningún lugar de la tierra. En esta sociedad el ciudadano como individuo particular deja de pertenecer a una esfera privada de la existencia para entregarse públicamente al Estado que determina su formación integral. La subordinación del individuo al Estado sería total y absoluta, controlando desde el nacimiento de los hijos

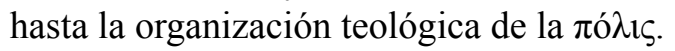

El principal cimiento sobre el que asientan los ideales utópicos de Platón se basan en una correcta educación de los ciudadanos desde el mismo nacimiento. El poeta, el artista o el sofista tradicional estaban totalmente desautorizados para realizar la tarea fundamental del Estado. La enseñanza no podía recaer en falsos intelectuales que no tenían una idea acertada de la verdad o la belleza, que malcriaban a los jóvenes con falsas historias de mitos, y que difundían un mensaje erróneo de la realidad. La justicia totalitaria del Estado platónico frenaba y bloqueaba cualquier atisbo de innovación de sus instituciones ${ }^{32}$, tal y como ocurría en Esparta o Egipto con el inmovilismo de sus instituciones ${ }^{33}$. Uno de los instrumentos de los cuales se valía el legislador o el guardián para mantener la disciplina o el orden era el de la censura. Toda innovación cultural, religiosa, festiva o política debía ser seccionada rápidamente. Pero Platón era conocedor

\footnotetext{
${ }^{30}$ POPPER, K. R. The Open Society and Its Enemies. Londres: Routledge, 1945.

31 VIDAL-NAQUET, P. Le chasseur noir: Formes de pensée et formes de societé dans le monde grec. Paris: Editions La Decouverte, 1981. p. 317; DUBOIS, P. The History of the Impossible: Ancient Utopia. ClassicalPhilol, v. 101, n. 1, 2006, p. 1-15; STECKER, R. Plato's Expression Theory of Art. JAesthetEduc, v. 26, n. 1, 1992, p. 47-52.

32 DEMOS, R. Was Plato a Fascist? Classical Weekly, v. 35, oct.1941/jun.1942, p. 243.

33 DAVIS, W. M. Plato on Egyptian Art. JEgyptArchaeology, v. 65, 1979, p. 121-127; GODEL, R. Platon a Héliopolis d'Égypte. Paris: Les Belles Lettres, 1956; DONOVAN, B. R. The Do-It-Yourself in Plato's Republic. AMJPhilology, v. 124, n. 1, 2003, p. 1-19.
} 
de que no podía existir una sociedad carente de arte o poesía, entonces ¿quién sería el encargado de difundir el mensaje artístico o poético? Platón entonces nombra a los guardianes como los encargados de salvaguardar la salud cultural del Estado.

Los guardianes de la República debían ejercitarse, como

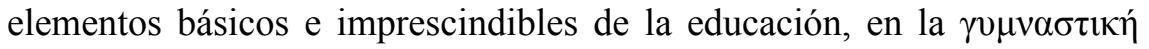

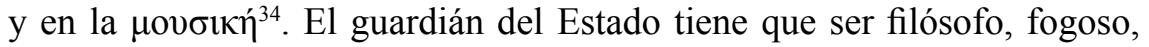
rápido y fuerte tal y como lo define en $376 \mathrm{c}-\mathrm{d}$, y además "la $\pi \alpha 1 \delta \varepsilon i ́ \alpha$ del guardián se rige en todos y cada uno de sus momentos por el principio del discernimiento" 35 . Los guardianes tenían la misión de controlar y censurar a los $\mu v \theta$ o $\pi$ otoí, a los "hacedores de mitos" (Rep. 378-e7), puesto que los niños debían educarse en historias válidas y aptas para su formación en la obtención de la $\sigma \omega \varphi \rho o \sigma u ́ v \eta$, de la "prudencia, cordura, sensatez", y de su obediencia a la autoridad establecida que velaba sobre el control de sus deseos en la sociedad cerrada. Resulta sorprendente que en la descripción que hace Platón de los guardianes falten elementos relacionados con lo guerrero o belicoso, ya que las enseñanzas gimnásticas y musicales eran su principal prioridad. Los guardianes son víctimas de un sistema opresor, autoritario, que limita las libertades individuales por medio de un sistema pedagógico dirigido y censurado. Los libros II-III de la República muestran algunos paralelismos con los libros II-VII de las Leyes, pues en ambos se censura la poesía como inmoral, pero se aceptaría si llegara a adaptarse a los principios morales reclamados por el Estado ${ }^{36}$. La necesidad de verdad y de moral debía de ser una cualidad indiscutible para los narradores de historias (Rep. 399c-d), ya que el orden y la armonía en el alma se consiguen mediante un uso justo y equilibrado de las pasiones (Rep. 400d-401d).

Finalmente Platón, para poder encontrar una solución real, decide aceptar algunos tipos de imitación en su Estado. Serán aquellos artistas miméticos capaces de representar el buen carácter (Rep. 401b1-d3) los

\footnotetext{
34 NETTLESHIP, R. L. Lectures on the Republic of Plato. London: MacMillan, 1937, p. 99 y ss., relaciona la $\mu$ ovбıкท́ directamente con la literatura y explica las causas.

35 MARZOA, F. M. Ser y diálogo: Leer a Platón. Madrid, 1996. p. 70.

36 PARTEE, M. H. Plato's Banishment of Poetry. JAesthetArtCritic, v. 29, n. 2, 1970, p. 209-222.
} 
que tengan cabida en la sociedad platónica. En el libro III de la República Platón asume la importancia del arte en la sociedad, especialmente de la poesía $^{37}$. En 377d7-e3, destaca que lo primero en censurar son las mentiras innobles, cuando los dioses y los héroes son mal representados a través de un lenguaje falso, igual que en un pintor que no pinta lo que se ha propuesto pintar. La censura y la supervisión de las artes miméticas por parte de los guardianes apuntan a la $\pi \alpha 1 \delta \varepsilon i ́ \alpha$, puesto que Platón ejemplifica las futuras expulsiones de los artistas con ejemplos de poetas contemporáneos que perjudican la formación de la juventud griega, como Homero, Hesíodo o Esquilo ${ }^{38}$. En República 395c, donde Sócrates concluye que los guardianes ${ }^{39}$, denominados como "artesanos de la libertad del Estado en sentido estricto", no deben imitar cualquier cosa que resulte inconveniente; en el caso de hacerlo, correspondería que imitasen ya desde niños los tipos que le son apropiados, como los valientes, moderados, piadosos o libres ${ }^{40}$. En estos fragmentos del texto, el infinitivo $\mu \mu \varepsilon \tilde{\sigma} \sigma \theta \alpha$ es utilizado cuando trata de las melodías y ritmos adecuados en la representación musical ${ }^{41}$. En el libro III de República, los modos musicales, los instrumentos utilizados y los ritmos son considerados en relación directa con la formación del carácter de los ciudadanos ${ }^{42}$.

37 DYSON, M. Poetic Imitation in Plato Republic 3. Antichton, v. 22, 1988, p. 42-53.

38 En el ataque contra los poetas imitativos Platón siempre utiliza como ejemplos a los poetas que le parecían más trascendentes y a los que creía como mejores, especialmente a Homero y a los grandes trágicos del siglo V a.C., y no a la literatura considerada como "inferior", ver URMSON, J. O. Plato and the Poets. Plato on Beauty, Wisdom and the Arts, p. 125-137.

39 TARRANT, D. Plato as Dramatist. JHellenicStud, v. 75, 1955, p. 82-89. Ha identificado esos fragmentos que tienen una fuente dramática: Charm. 162d, Eutidemo 276b, Crat. 425d, Rep. 580b, Polit. 260c, 303c, Phaed. 115a, Prot. 327d. Además en algunos pasajes las metáforas sugieren el paralelo entre la asamblea y la audiencia del teatro, tratando el diálogo mismo como un drama, como en: Symp. 194a-b, Prot. 315b, Crit. 108b, Phil. 50b, Theaet. 193c. Una de las conclusiones a las que llega el autor es que todas las obras de Platón, excepto Apol. y Epist., están escritas siguiendo un guión dramático.

40 SÖRBOM. op.cit. p. 117-128. "Plato: the Second and Third Books of the Republic".

41 Para una visión de la música contemporánea a Platón, y de la relación que se establece entre ambos: SCHUHL, P. M. Platon et la musique de son temps. In: Études platoniciennes. Paris: Puf, 1960, p. 100-112.

${ }^{42}$ LIPPMAN, E. A History of Western Musical Aesthetics. Londres: University of Nebraska 
Las Leyes constituye el ejemplo sobre el que se basan las afirmaciones que apuntan a una actitud más consciente de Platón en sus últimos diálogos respecto al arte, mientras que en los primeros, conocedor de la dificultad de la censura sobre el arte, se presenta con una actitud más agresiva y extremista ${ }^{43}$. En esta obra se encuentra la visión más relajada y sosegada de Platón hacia las artes, puesto que ya no se encuentra en un Estado Ideal, sino en una sociedad real donde debe primar el bienestar: los jóvenes son educados para tener sentimientos justos, y los de mayor edad pueden gozar y descansar del recreo en el arte de las Musas, (653d). En las Leyes, (801c), un Platón ya anciano, que no se empeña ya en la construcción de un Estado Ideal sino en la creación de un bienestar real, establece que el poeta no debe componer nada que no se adapte a la concepción de "lo justo" por parte del Estado, y además no debía exhibir sus obras hasta que no haya sido aprobado por los jueces y los guardianes de la ley. Es fácil citar en este momento el moderno concepto de "censura"44. A partir de este modelo fija las pautas de la censura, o de la subordinación del arte al bien, y debido a la claridad con la que concebía el peligro de una excesiva libertad, reaccionó de manera tajante y extrema enunciando la primera teoría sobre la censura en el arte. Es precisamente en este punto donde reside la mayor modernidad de la teoría platónica: supo ver perfectamente el problema del pueblo, adentrarse en él y establecer los principios y las posibles soluciones.

Aún hoy en día constituye un argumento de actualidad, y siempre lo seguirá siendo, el grado de implicación del arte en la sociedad y la repercusión de los mensajes lanzados a través de los actuales "mass media", antiguas representaciones poéticas y teatrales, en la conciencia de los ciudadanos de las modernas $\pi$ ó $\lambda \varepsilon ı \varsigma$ y en la formación de un espíritu cívico ético y

Press, 1992. p. 10-11.

43 VERSÉNYI, L. The Cretan Plato. RevMetaphysics, v. 15, n. 1, 1961, p. 67-80; MORROW, G. R. Plato's Cretan City. Princeton: Princeton University Press, 1960; BERTRAND, J-M. De l'écriture à l'oralité: Lectures des Lois de Platon. Paris: Publications de la Sorbonne, 1999.

44 Tal y como ya hizo GIL, L. Censura en el mundo antiguo. Madrid: Alianza Editorial, 1961. En la parte primera, capítulo II, p. 77-105 se refiere a Platón al tratar "La opinión de los filósofos". 
moral. Las artes miméticas en Leyes, entendiendo por "artes miméticas" el conjunto de música, danza, poesía, drama, pintura y escultura, pueden tener formas maravillosas y no sólo centrarse en la representación de las apariencias $^{45}$. Ya que, para Platón, la educación no consiste simplemente en la adquisición de conocimientos, sino que también versa acerca del aprendizaje en el amor y en las buenas costumbres, (653b). Los libros IIIII de la República y, especialmente, los libros II-VII de Leyes $^{46}$, reafirman la visión platónica del arte a la luz de la educación, de la moral y de la política, haciendo una revisión de las artes para cambiar el modelo social y ético de su tiempo, a partir del análisis del poder psicológico del arte y de las ideas comunicadas por el artista al público ${ }^{47}$. La idea de arte propuesta, e impuesta, por Platón debía cumplir con la verdad, República (484), y con la benevolencia, República (379), en la educación de los más jóvenes.

Hasta este punto se ha impuesto una visión jerarquizada de las artes, en la que tan sólo pueden ser admitidas aquellas que, de acuerdo con su misión mayéutica, pueden cumplir una labor social pedagógica. Si no tuviéramos el inicio del libro X de la República la visión de las artes por parte de Platón no hubiese sido tan polémica y contradictoria. Sorprende el tratamiento ${ }^{48}$, además del tono más agresivo, que utiliza en este libro en comparación con los libros II-III ${ }^{49}$. Además, también llama la atención que Platón vuelva sobre un tema que ya había tratado con anterioridad, si bien está absolutamente justificado que Platón examine una vez más, sobre esta base (la base de la teoría de las Ideas), el problema de la poesía.

45 SCHIPPER, E. W. Mimesis in the Arts in Plato's Laws. JAesthetArtCritic, v. 22, n. 2, 1963, p. 199-202.

46 Según el criterio convencional se encuentran ampliamente descritos en KRÜGER, J. (Ed.). Ästhetik der Antike. Berlin-Weimar: Laufbau-Verlag, 1964. p. 514 y ss.

47 GILBERT, K. The Relation of the Moral to the Aesthetic Standard in Plato. PhilosRev, v. 43, n. 3, 1934, p. 279-294.

48 BABUT, D. Paradoxes et énigmes dans l'argumentation de Platon au livre X de la République. In: BRUNSCHWIG, J. (org.). Historie et Structure. Hommages á Victor Goldschmidt. Paris: Vrin, 1985, p. 133.

49 Para una justa interpretación de la relación entre los distintos diálogos platónicos CORLETT, J.A. Interpreting Plato's Dialogues. $C Q$, New Series, v. 47, n. 2, 1997, p. 423437. 
La opción del libro X como coda significa entender el contenido de dicho libro como un apéndice respecto al contenido anteriormente tratado en los demás libros.

\section{La selección de los artistas en República 595a}

El repentino cambio del comienzo del libro X de la República se justifica de inmediato, ya que se menciona la división del alma como argumento tratado con anterioridad, pudiendo ahora acometer la lucha contra la poesía, enemiga número uno de la razón, con el sólido argumento del alma ya asentado y con la Teoría de las Ideas expuesta ${ }^{50}$. Personalmente, aquí encuentro el principal argumento para justificar la unión del libro $\mathrm{X}$ con el resto del diálogo. No se puede entender las afirmaciones tan duras de Platón sin saber en qué basa esas gravísimas acusaciones.

La mayoría de los argumentos platónicos contra las artes se encuentra en este libro X: en 595a-602b, a partir de una nueva definición de $\mu$ í $\eta \sigma 1 \varsigma$, resalta la insuficiencia respecto a la realidad y al conocimiento de todas las artes miméticas, produciéndose la expulsión de todos los artistas. La teoría de las Ideas, desarrollada más ampliamente en los libros V-VII, es usada para demostrar la naturaleza de la imitación. Entre 602c-605c describe la principal arma de la poesía; aquella que afecta a la parte más corruptible de la mente humana produciendo devastadoras consecuencias, ya que el alma puede percibir la verdad sólo a través del pensamiento, no de los sentidos. La parte irracional del alma sólo puede ver apariencias. Por último, (605c-608b): el arte como fuente de una moralidad negativa y perjudicial en la sociedad, pues los mensajes asimilados de las historias son tan variados y arbitrarios como el ingenio y el poder del escritor y la receptividad que la audiencia permiten ${ }^{51}$.

50 GILBERT, A. H. Did Plato Banish the Poets or the Critics? StudPhilol, v. 36, 1939, p. 1-19; Según WHITE, N. P. A companion to Plato's Republic. Oxford: Hackett, 1979, dentro de República se encuentran en ocasiones digresiones que no siguen un orden natural, encontrando el máximo ejemplo en 608b-608c cuando pasa repentinamente de hablar sobre la poesía a tratar directamente el tema de la inmortalidad del alma y sus respectivas consecuencias.

51 SKILLEN, A. Fiction Year Zero: Plato's Republic. BritJAesthet, v. 32, n. 3, 1992, p. 
Mientras que en el libro III se propone la expulsión de algunos poetas imitativos, de aquellos que realizaban un uso profundo e indiscriminado de la caracterización dramática, en el libro X (595a) todos son expulsados. En 392d, había dividido la poesía en representativa, sobre todo refiriéndose a la tragedia y a la comedia, y no representativa, ya que ciertas poesías representativas son consideradas como poco aconsejables para la educación de los jóvenes y de los guardianes, (398a). En esta línea interpretativa, el punto 595a continúa con la antigua división realizada en los libros II-III; el ataque de Platón se centra en la poesía representativa

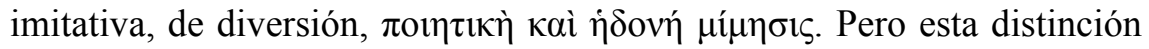
entre la imitación utilizada en el libro III y la del libro X es insuficiente para resolver el aparente conflicto entre ambos libros; pues mientras en el libro III se permiten el género del ditirambo y poemas en los que los hombres honorables son enaltecidos, en el libro $\mathrm{X}$ se excluye, aparentemente, a todo tipo de artista imitativo. Así, la función del arte es la de impartir conocimientos, pero como se muestra incapaz de hacerlo, debe introducir a los jóvenes en la armonía del carácter y familiarizarlo con las rectas opiniones $^{52}$. De esta manera, es necesaria la lectura del libro X como coda o remate final a todo el discurso anterior de la República para encontrar sentido a las conclusiones finales de Platón. La última prueba de este argumento se encuentra en $607 \mathrm{a}$, cuando definitivamente conserva ciertas clases de poesía no representativa al admitir que sólo deberán admitirse en nuestro Estado los himnos a los dioses y las alabanzas a los hombres buenos.

Existen dos tríadas fundamentales en el libro X: una de ellas referida a los hacedores, (596-598), que consta de Dios-artesano-artista; y la tríada de habilidades, (600-602), formada por usar el objeto-hacer el objeto-imitar el objeto ${ }^{53}$. Tres grados alejados de la verdad se encuentra el

201-208.

52 TATE, J. Plato and Imitation. $C Q$, v. 26, n. 3/4, 1932, p. 161-169; TATE, J. Imitation in Plato's Republic. $C Q$, v. 22, n. 1, 1928, p. 16-23; OATES, W. J. Plato's Philosophical Creative Artist. Classical Weekly, v. 35, oct. 1941/jun. 1942, p. 247; MASTRANGELO, M.; HARRIS, J. The Meaning of Republic 606a3-b5. CQ, v. 47, n. 1, 1997, p. 301-305; HALL, R. W. Plato's Theory of Art: A Reassessment. JAesthetArtCritic, v. 33, n. 1, 1974, p. $75-82$.

${ }_{53}$ SCHWEITZER, B. Zur Kunst der Antike: Ausgewählte Schriften. Band I. Tübingen: Ernst Wasmuth, 1963, p. 53. 
arte imitativo. La poesía se convierte en un peligro intelectual para todos aquellos que no tienen un antídoto contra ella, ya que en el caso de los objetos de tercer grado no hay forma de distinguir la verdad del error ${ }^{54}, \mathrm{y}$

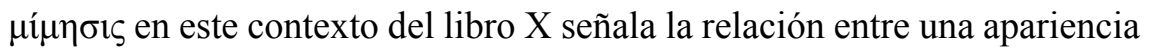
y la realidad de esa apariencia en cuestión. De esta manera, otro atributo peyorativo y negativo se le añade al arte mimético; el de "estar alejado tres

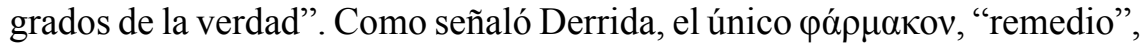
contra este tipo de poesía imitativa es el conocimiento de las cosas que realmente son $^{55}$.

\section{La realidad del arte y el conservadurismo platónico}

El discurso teórico de Platón tiene un estricto paralelismo en el arte de su época. Su admiración por el arte arcaico o egipcio, alejan al filósofo de las novedades artísticas que se están realizando en la Grecia de su tiempo ${ }^{56}$. Los testimonios directos sobre artistas son escasos en la obra de Platón, y sus citas sobre las diferentes prácticas artísticas están en ocasiones encerradas en una metáfora ${ }^{57}$, o por analogía en comparaciones entre el discurso escrito y las obras pictóricas de los pintores. En Político (299d-e), se aprecia la complejidad que supone trazar una línea única de

54 COLLingWOOD, R. G. Plato's Philosophy of Art. Mind, v. 34, n. 134, 1925, p. 154172 .

55 DERRIDA, J. Dissémination. Paris: Seuil, 1972, p. 237.

${ }^{56}$ DEMAND, N. Plato and the Painters. Phoenix, v. 29, 1975, p. 1-20; SCHUHL, P. Platon et l'art de son temps. Paris, 1933; RUMPF, A. Classical and Post-Classical Greek Paintig. JHellenicStud, v. 67, 1947, p. 10-21; STEVEN, R. G. Plato and the Art of His Time. CQ, v. 27, n. 3/4, jul./oct.1933, p. 149-155.

57 LOUIS, P. Les métaphores de Platon. Paris, 1945. Cita los fragmentos metafóricos en

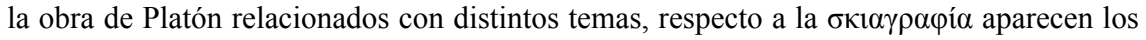
siguientes, pp. 210: Phaed. 69b, Rep. 365c, 583b, 586b, Theaet. 208e, Parm. 165c, Crit.

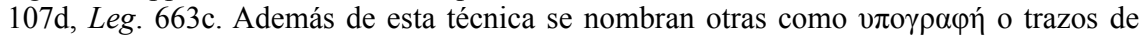
pintura en esbozo, en Rep. 501a,504d, 548d, Theaet. 171e, Leg. 711b, 734e, 737d, 934c;

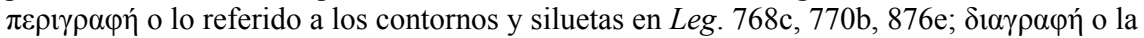
delineación plana de las figuras en Leg. 778a-c. Sin embargo, las referencias a la escultura son bastantes menos numerosas y se centran en comparaciones de Sócrates con estatuas de Sileno (Symp. 215b, 216d, 221d) o alusiones a las obras del legendario escultor Dédalo (Euthyphr. 11b, 15b, Men. 97d, 98a). 
pensamiento o de opinión en Platón respecto al arte, después de plantear la posibilidad de establecer un canon fijo para todo tipo de artistas imitativos, ya que está bien claro que todas las artes nos quedarían por completo destruidas y ya nunca más podrían nacer en el futuro, a causa de esa ley que interferiría toda búsqueda. De ahí que la vida, que ya ahora es difícil, se volvería entonces absolutamente intolerable.

Especialmente interesante y sorprendente para Schweitzer resulta la ausencia de grandes artistas del s.IV a.C., como Escopas o Praxíteles ${ }^{58}$, y llega a proponer la hipótesis de que el verdadero enemigo al cual se refiere Platón en el libro X de la República es la Escuela de Sición, que en 369 a.C. fue conquistada y sirvió de base para los ejércitos tebanos, en el exitoso ataque que esta ciudad hizo a la Liga del Peloponeso. Durante este periodo, Sición alcanzó su cénit como centro artístico: sus escuelas de pintura alcanzaron fama por Eupompo, y atrajeron a los grandes maestros Pánfilo y Apeles, que era jonio pero estudió allí y llegó a ser pintor de cámara de Alejandro Magno. La escuela de los pintores llamados sicionios ejerció una notable influencia, y de algunos de sus artistas nacieron las principales novedades del estilo pictórico, como los diseños florales y de follaje. Su tradición escultórica alcanzó el máximo nivel con Lisipo, natural de Sición, y sus alumnos. El trabajo de Pánfilo en Atenas fue conocido hacia el 388 a.C., y en él se encuentran las primeras evidencias de la técnica ilusionista de la

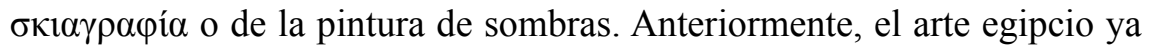
había mostrado que el uso de unos cánones matemáticos no implicaba el uso de perspectiva ${ }^{59}$, y los griegos retomaron este antiguo camino ya recorrido.

Una de las técnicas que aparecen citadas en la obra de Platón es la бкıаүрафía ${ }^{60}$, que consistía en realizar los contornos y figuras de los

58 SCHWEITZER, B. Platon un die bildende Kunst der Griechen. Tübingen: Niemeyer, 1953. El gran mérito del libro es la doble lectura que realiza sobre Platón y el arte de su tiempo: por una parte destaca la crítica violenta sobre el arte ilusionista como fuente de un elemento emocional perjudicial para la educación, pero por otra parte resulta indudable la influencia que el filósofo tuvo para compartir el objetivo de los artistas de entrar en las mentes humanas.

59 Esta hipótesis la defienden SCHWEITZER y BYVANCK, A. W. Platon et l'art grec. Bulletin Antieke Beschaving, v. 30, 1955, p. 35-39.

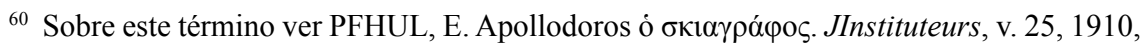


cuerpos mediante un sombreado que los dotaba de profundidad y relieve, República (523b). El concepto en sí mismo denota un interés por los efectos de la luz, como confirma Plinio $(H N, 35.29)$. Platón señaló la cercanía entre esta nueva pintura ilusionista y el movimiento cultural de los sofistas, contraponiendo a la estética de la Imitación platónica la estética de la Ilusión gorgiana; pero, ¿en qué orden situamos esta oposición? Para Schuhl, si Platón se muestra tan severo con las artes es "seguramente porque les reconoce - por haberlas experimentado, sin duda - la influencia irracional que les atribuía Gorgias... y para contrarrestarla recurre al idealismo moral y matemático; contra los artificios de los ilusionistas apela a la medida, al número, al peso" 61 .

Aunque fue en la época contemporánea de Platón cuando la revolución griega en el arte llegaba a su mayor expresión, mediante un verdadero arte de vanguardia ${ }^{62}$ : los escorzos y los efectos de modelado a través del uso del claroscuro abrían una nueva etapa en el arte griego, y Platón tenía razón al sentir que algo se había sacrificado en aquel cambio: la función intemporal de la imagen poderosa, el faraón dominando para siempre a sus enemigos, tenía que abandonarse a favor de un imaginario fugaz instante, que fácilmente podría tentar a un artista a la trivialidad.

En Protágoras (318c), Platón cita a uno de los artista más destacados de su época, reconocido públicamente y admirado por encima del resto de los pintores, se trata de Zeuxis de Heraclea ${ }^{63}$, también citado en Gorgias 453c, y que comenzó a pintar sobre 452 a.C. En la totalidad de la obra de Platón se pueden encontrar diferentes posturas sobre la pintura: en Ion, Gorgias, Crátilo, República II-VI, aparece el pintor como un miembro

p. 12-28; JInstituteurs, v. 27, 1912, p. 227-231; SCHÖNE, R. Skiagrafia. JInstituteurs, v. 27, 1912, p. 19-23.

61 SCHUHL, P. op.cit. p. 72.

62 Así la denomina GOMBRICH, E. H. Art and Illusion: A Study in the Psychology of Pictorial Representation. Washington: Phaidon Press, 1959. "Reflexiones sobre la revolución griega", p. 99-125.

${ }^{63}$ OVERBECK, T. Die Antiken Schriftquellen zur Geschichte der bildenden künste bei den Griechen. Hildesheim: Georg Holm, 1959. Fundamental para comprobar las fuentes tanto en griego como en latín de los artistas y poetas. 
respetable de la comunidad de los creadores que poseen una $\tau \dot{\varepsilon} \chi v \eta$; en Fedón, República VII-X, Teeteto, Parménides, Fedro, se destaca el poder

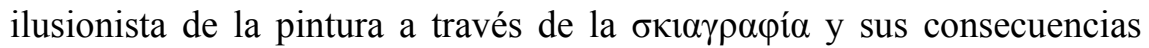
emocionales sobre los espectadores; y en Fedón, Sofista y Leyes persiste la visión negativa de la pintura ilusionista.

\section{Conclusiones}

Este arte que Platón contemplaba era, siguiendo su discurso basado en el concepto de mayéutica incapaz de satisfacer los deseos platónicos, no podía sacar de las sombras ni arrojar luz sobre el mundo de la opinión, no se acercaba de manera ontológica a la verdad ni a la belleza ni al bien. Así, la afirmación al comienzo del libro X de la República se refiere a todos los artistas imitativos que no siguen lo dicho posteriormente en 607a, sólo deberán admitirse en nuestro Estado los himnos a los dioses y las alabanzas a los hombres buenos. La labor de la filosofía de Platón es la de desvincular al arte de la educación y de la filosofía, de cualquier labor legislativa cívica o pedagógica. En consecuencia, Platón niega al arte de su tiempo estas cualidades mínimas que requieren las técnicas miméticas para poder ser consideradas aptas para la juventud. La pintura de sombras,

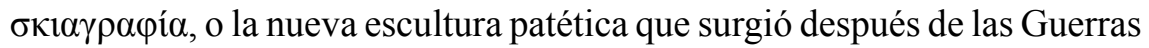
del Peloponeso, no mostraban la verdad en lo alto de la metáfora del libro VII de la República, la esencia de las cosas y de los seres seguía estando alejada de estas representaciones materiales. Es necesario recordar que Platón, en muchos puntos de su obra, trata los temas relacionados con el arte y los artistas desde una perspectiva casi cómica y, que el concepto de juego, $\pi \alpha 1 \delta i ́ \alpha$, es significativo de la actitud que tomaba respecto a estos temas. De igual manera que el padre debe controlar y organizar los juegos que un niño realiza en su infancia, así el gobernante filósofo, guiado por la razón, tiene que censurar y establecer la rectitud necesaria en el arte que los artistas y el resto de los ciudadanos no han sido capaces de imponerse, al no haber completado el proceso de $\pi \alpha 1 \delta \varepsilon i ́ \alpha$. En la contundencia de la respuesta de Platón se encuentra la importancia del problema planteado. 


\section{Referências}

AUERBACH, E. Mímesis: La representación de la realidad en la literatura occidental. México: Fondo de Cultura Económica, 1987.

BABUT, D. Paradoxes et énigmes dans l'argumentation de Platon au livre $\mathrm{X}$ de la République. In: BRUNSCHWIG, J. (org.). Historie et Structure. Hommages á Victor Goldschmidt. Paris: Vrin, 1985.

. Sur la notion d'Imitation dans les doctrines esthétiques de la Gréce classique. REG, n. 98, p. 72-92, 1985.

BAEUMLER, A. Ästhetik. Berlin: Oldenbourg, 1934.

BERTRAND, J-M. De l'écriture à l'oralité: Lectures des Lois de Platon. Paris: Publications de la Sorbonne, 1999.

BOYD, J. D. The Function of Mimesis and Its Decline. Cambridge: Harvard University Press, 1968.

BYVANCK, A. W. Platon et l'art grec. Bulletin Antieke Beschaving, n. 30, p. 35-39, 1955.

COLLINGWOOD, R. G. Plato's Philosophy of Art. Mind, v. 34, n. 134, p. 154-172, 1925.

CORLETT, J. A. Interpreting Plato's Dialogues. $C Q$, New Series, v. 47, n. 2, p. 423-437, 1997.

DAVIDSON, J. Greek Drama: image and audience(s). BIClassStud, v. 48, p. 1-15, 2005.

DAVIS, W. M. Plato on Egyptian Art. JEgyptArchaeology, v. 65, p. 121$127,1979$.

DE ANGELI, S. Mimesis e Techne. QUCC, n. 57, p. 27-45, 1988.

DELATTE, A. Les conceptions de l'enthousiasme chez les philosophes présocratiques. Paris: Les Belles Lettres, 1934.

DELEUZE, G. Platón y el simulacro. Lógica del sentido. Barcelona: Paidós, 2005.

DEMAND, N. Plato and the Painters. Phoenix, v. 29, p. 1-20, 1975.

DEMOS, R. Was Plato a Fascist? Classical Weekly, v. 35, p. 243, oct.1941/ jun. 1942. 
DERRIDA, J. Dissémination. Paris: Seuil, 1972.

DONOVAN, B. R. The Do-It-Yourself in Plato's Republic. AMJPhilology, v. 124, n. 1, p. 1-19, 2003.

DODDS, E. R. The Ancient Concept of Progress and other Essays on Greek Literatura and Belief. Oxford: Clarendon Paperbacks, 1973.

DUBOIS, P. The History of the Impossible: Ancient Utopia. ClassicalPhilol, v. 101, n. 1, p. 1-15, 2006.

DYSON, M. Poetic Imitation in Plato Republic 3. Antichton, n. 22, p. 4253, 1988.

EDMONDS, R. G. Socrates the Beautiful: Role Reversal and Midwifery in Plato's Symposium. TAMPhilolAssoc, n. 130, p. 261-285, 2000.

FLASHAR, H. Der Dialog Ion als Zeugnis platonischer Philosopie. Berlin: Akademie-Verlag, 1958.

. Die Klassizistische Theorie der Mimesis. Le Classicisme a Rome, n. 25, p. 79-111, 1978.

GIL, L. Censura en el mundo antiguo. Madrid: Alianza Editorial, 1961.

GILBERT, A. H. Did Plato Banish the Poets or the Critics? StudPhilol, v. 36, p. 1-19, 1939.

GILBERT, K. The Relation of the Moral to the Aesthetic Standard in Plato. PhilosRev, v. 43, n. 3, p. 279-294, 1934.

GODEL, R. Platon a Héliopolis d'Égypte. Paris: Les Belles Lettres, 1956. GOLDEN, L. Plato's Concept of Mimesis. BritJAesthet, v. 15, n. 2, p. 118$131,1975$.

GOMBRICH, E. H. Art and Illusion: A Study in the Psychology of Pictorial Representation. Washington: Phaidon Press, 1959.

GRIFFIN, J. The Social Function of Attic Tragedy. CQ, v. 48, n. 1, p. 39$61,1998$.

GRISWOLD, C. The Ideas and the Criticism of the Poetry in Plato's Republic, Book 10. JHistPhilos, v. 19, n. 2, p. 135-150, 1981.

HALL, R. W. Plato's Theory of Art: A Reassessment. JAesthetArtCritic, v. 33, n. 1, p. 75-82, 1974. 
HALLIWELL, S. The Aesthetics of Mimesis: Ancient Texts and Modern Problems. Princeton: University Press, 2002.

HUBER-ABRAHAMOWICZ, E. Das Problem der Kunst bei Platon. Winterthur: Keller, 1954.

KEULS, E. Plato and Greek Painting. Leiden: Brill, 1978.

KOLLER, H. Die Mimesis in der Antike: Nachahmung, Darstellung, Ausdruck. Berna: Francke, 1954.

KRÜGER, J. (Ed.). Ästhetik der Antike. Berlin-Weimar: Laufbau-Verlag, 1964.

LADRIERE, C. The Problem of Plato's Ion. JAesthetArtCritic, v. 10, n. 1, p. 26-34, 1951.

LESHER, J. H. The Afterlife of Plato's Symposium. ClassicalJ, v. 100, n. 1, p. 75-87, 2004.

LIMINTA, M. T. Il problema della Belleza in Platone: Analisis e interpretazioni dell "Ippia Maggiore”. Milán: Vita e Pensiero, 1998.

LIPPMAN, E. A History of Western Musical Aesthetics. Londres: University of Nebraska Press, 1992.

LYONS, J. Structural Semantics: An Análisis of Part of the Vocabulary of Plato. Oxford: Blackwell, 1972.

MASTRANGELO, M.; HARRIS, J. The Meaning of Republic 606a3-b5. $C Q$, v. 47, n. 1, p. 301-305, 1997.

MCKEON, R. Literary Criticism and the Concept of Imitation in Antiquity. ModPhilology, v. 34, n. 1, p. 1-35, 1936.

MORAUX, P. La "Mimesis" dans les théories anciennes de la danse, de la musique et de la poésie. $L E C$, v. 23, n. 1, p. 3-13, 1955.

MARZOA, F. M. Ser y diálogo: Leer a Platón. Madrid: Istmo, 1996.

MÁSMELA, C. Dialéctica de la imagen: Una interpretación del Sofista de Platón. Barcelona: Antropos Editorial, 2006.

MOORE, J. D. The Dating of Plato's Ion. GreekRomanByzantin, v. 15, n. 4, p. 421-439, 1974.

MORGAN, M. The Continuity Theory of Reality in Plato's Hippias Major. JHistPhilos, v. 21, n. 2, p. 133-158, 1983. 
MORROW, G. R. Plato's Cretan City. Princeton: Princeton University Press, 1960.

MURRAY, P. Plato on Poetry: Ion; Republic 376e-398b9; Republic 595608b10. Cambridge: University Press, 1996.

NETTLESHIP, R. L. Lectures on the Republic of Plato. London: MacMillan, 1937.

NOTOMI, N. The Unity of Plato's Sophist: Between the Sophist and the Philosopher. Cambridge: University Press, 1999.

OATES, W. J. Plato's Philosophical Creative Artist. Classical Weekly, v. 35, p. 247, oct. 1941/jun. 1942.

OVERBECK, T. Die Antiken Schriftquellen zur Geschichte der bildenden künste bei den Griechen. Hildesheim: Georg Olms 1959.

PARTEE, M. H. Plato's Banishment of Poetry. JAesthetArtCritic, v. 29, n. 2, p. 209-222, 1970.

PANOFSKY, E. Idea: Contribución a la historia de la teoría del arte. Madrid: Catedra, 1977.

PHILIP, J. A. Mimesis in the Sophistes of Plato. TAMPhilolAssoc, v. 92, p. 453-468, 1961.

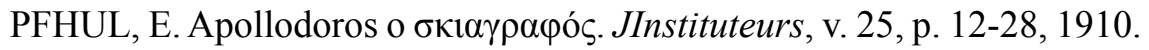
POPPER, K. R. The Open Society and Its Enemies. Londres: Routledge, 1945.

RUMPF, A. Classical and Post-Classical Greek Paintig. JHellenicStud, v. 67, p. 10-21, 1947.

SCHIPPER, E. W. Mimesis in the Arts in Plato's Laws. JAesthetArtCritic, v. 22, n. 2, p. 199-202, 1963.

SCHÖNE, R. Skiagrafia. JInstituteurs, v. 27, p. 19-23, 1912.

SCHUHL, P. M. Platon et la musique de son temps. In: Études platoniciennes. Paris: Puf, 1960. p. 100-112.

SCHWEITZER, B. Platon un die bildende Kunst der Griechen. Tübingen: Walter de Gruyter, 1953.

. Zur Kunst der Antike: Ausgewählte Schriften. Band I. Tübingen: Ernst Wasmuth 1963. 
SKILLEN, A. Fiction Year Zero: Plato's Republic. BritJAesthet, v. 32, n. 3, p. 201-208, 1992.

SOBREVILLA, D. La Estética de la Antigüedad: Estudios sobre lo bello y el arte en el pensamiento de Platón, Aristóteles, Cicerón y Plotino. Caracas: Universidad Carabobo, 1981.

SOLMSEN, F. Parmenides and the Description of Perfect Beauty in Plato's Symposium. AMJPhilology, v. 92, n. 1, p. 62-70, 1971.

SÖRBOM, G. Mimesis and Art: Studies in the Origin and Early Development of an Aesthetic Vocabulary. Uppsala: Svenska Bökforlaget, 1966.

STECKER, R. Plato's Expression Theory of Art. JAesthetEduc, v. 26, n. 1, p. 47-52, 1992.

STEVEN, R. G. Plato and the Art of His Time. $C Q$, v. 27, n. 3/4, p. 149155, jul./oct.1933.

TARRANT, D. Plato as Dramatist. JHellenicStud, v. 75, p. 82-89, 1955.

TATE, J. Plato and Imitation. CQ, v. 26, n. 3/4, p. 161-169, 1932. . Imitation in Plato's Republic. CQ, v. 22, n. 1, p. 16-23, 1928.

TIGERSTEDT, E. N. Furor Poeticus: Poetic Inspiration in Greek Literature before Democritus and Plato. JHistIdeas, v. 31, n. 2, p. 163-178, 1970.

URMSON, J. O. Plato and the Poets. In: MORAVCSIK, J.M.E.; TEMKO, P. (ed.). Plato on Beauty, Wisdom and the Arts. Totowa, N.J.: Rowman \& Littlefield, 1982. p. 125-137.

VERNANT, J. P. Image et apparence dans la théorie platonicienne de la mimesis. Journal de psycologie normale et pathologique, v. 62, p. 133160, 1975.

VIDAL-NAQUET, P. Le chasseur noir: Formes de pensée et formes de societé dans le monde grec. Paris: Editions La Decouverte, 1981.

VERDENIUS, W. J. Mimesis: Plato's Doctrine of artistic imitation and its meaning to us. Leiden: Brill, 1949.

. L'Ion de Platon. Mnemosyne, v. 11, p. 233-262, 1943.

. Der Begriff der Mania in Platons Phaidros. ArchivGeschPhilos, v. 44, n. 2, p. 133-151, 1962. 
VERSÉNYI, L. The Cretan Plato. RevMetaphysics, v. 15, n. 1, p. 67-80, 1961.

WALTER, J. Die Geschichte der Ästhetik im Alterum. Hildesheim: Georg Olms 1967.

WHITE, N. P. A companion to Plato's Republic. Oxford: Hackett, 1979.

WOODRUFF, P. Socrates and Ontology: The Evidence of the Hippias Major. Phronesis, v. 23, n. 2, p. 101-117, 1978.

Data de registro: $13 / 03 / 2013$ Data de aceite:20/11/2013 